\title{
Monoglyceride lipase mediates tumor-suppressive effects by promoting degradation of X-linked inhibitor of apoptosis protein
}

\author{
Renyan Liu $^{1,3} \cdot$ Xin Wang $^{1} \cdot$ Christopher Curtiss $^{2} \cdot$ M. Saeed Sheikh $^{1} \cdot$ Ying Huang $^{1}$
}

Received: 26 September 2019 / Revised: 14 April 2020 / Accepted: 16 April 2020 / Published online: 6 May 2020

(c) The Author(s), under exclusive licence to ADMC Associazione Differenziamento e Morte Cellulare 2020

\begin{abstract}
We have previously reported that Monoglyceride Lipase (MGL) expression is absent or reduced in various human malignancies and MGL-deficient mice develop tumors in multiple organs. Evidence also suggests MGL to be a tumor suppressor, however, the mechanisms underlying its tumor-suppressive actions remain to be investigated. Here, we report a novel function of MGL as a negative regulator of XIAP, an important inhibitor of apoptosis. We found that MGL directly interacted with XIAP and enhanced E3-ligase activity and proteasomal degradation of XIAP. MGL overexpression induced cell death that was coupled with caspase activation and reduced XIAP levels. N-terminus of MGL was found to mediate interactions with XIAP and induce cell death. MGL-deficient cells exhibited elevated XIAP levels and exhibited resistance to anticancer drugs. XIAP expression was significantly elevated in tissues of MGL-deficient animals as well as human lung cancers exhibiting reduced MGL expression. Thus, MGL appears to mediate its tumor-suppressive actions by inhibiting XIAP to induce cell death.
\end{abstract}

\section{Introduction}

Several lines of evidence from recent studies have demonstrated that Monoglyceride Lipase (MGL) plays an important role in controlling tumorigenesis [1-4]. Our previous studies have shown that MGL expression is frequently reduced in multiple human malignancies including tumors of the lung, colon, rectum, breast, stomach, and ovary [1, 2]. Information from Firehose/RSEM and Oncomine public databases also indicate that MGL (MGLL) mRNA expression is significantly

These authors contributed equally: Renyan Liu, Xin Wang

Edited by D. Vaux

Supplementary information The online version of this article (https:// doi.org/10.1038/s41418-020-0549-5) contains supplementary material, which is available to authorized users.

Ying Huang

huangy@upstate.edu

1 Department of Pharmacology, Upstate Medical University State University of New York, Syracuse, NY 13210, USA

2 Department of Pathology, Upstate Medical University State University of New York, Syracuse, NY 13210, USA

3 Present address: Department of Medical Oncology, Dana-Farber Cancer Institute, Bostone, MA 02215, USA reduced in multiple human malignancies $[5,6]$. We previously showed a significant portion of MGL-deficient mice exhibited lung adenocarcinoma and high-grade dysplasia [2]. MGL-deficient animals also exhibited spleen histiocytic sarcoma, ameloblastic adenoma, soft-tissue sarcoma, hepatoma and lymphoma at lower frequencies [2]. Depletion of MGL via RNAi or gene targeting significantly enhanced Akt and ERK phosphorylation in cancer cells and MGL-deficient lung tissues [1,2] and overexpression of MGL in cancer cells lacking endogenous MGL suppressed cancer cell growth [1]. However, the exact mechanisms via which MGL suppresses cancer cell growth and tumorigenesis remain to be further elucidated.

Induction of cell death is an important cellular function for eliminating the damaged cells with defective DNA and suppressing cancer formation [7-9]. X-linked inhibitor of apoptosis protein (XIAP) is a potent negative regulator of apoptosis [10]. It is an E3 ligase with three baculovirus inhibitory repeat (BIR) domains and a RING domain [11]. XIAP interacts with dimeric and activated caspases- 3 and -7 through the BIR-2 domain and prevents caspase- 3 from accessing its substrates [12-15]. Studies have also shown that XIAP degrades caspases-3 via the proteasomemediated mechanism [16]. Shiozaki et al. [17] showed that XIAP interacts with caspase- 9 via its BIR-3 domain; interaction of XIAP and caspase sequesters caspase- 9 in a monomeric state that blocks the activation and function of 
caspase-9. Overexpression of XIAP has been found in a variety of human cancers and was associated with anticancer drug resistance and poor survival of cancer patients [18]. However, the molecular mechanism(s) leading to XIAP overexpression in cancers remain unclear. The current study identifies MGL to be a novel negative regulator of XIAP and also demonstrates that modulation of XIAP is an important mechanism via which MGL mediates cell death and tumor suppression.

\section{Results}

\section{MGL-induced cancer cell death involves activation of apoptotic pathways}

We sought to investigate the mechanism(s) as to how MGL-deficiency leads to tumor development in animals. We found that exogenously expressed MGL in tumor cells lacking MGL caused significant cell death in human lung (H1299) and cervical (HeLa) cancer cells as well as in MGL-deficient mouse abdominal tumor cells (MADT) (Fig. 1a, b and Supplementary Fig. 1). Significant portion of RFP-MGL expressing H1299 and HeLa cells showed apoptotic nuclei (Fig. 1c, d). MGL overexpression also led to activation of caspases-3, 8, and 9 (Fig. 2a, b) and increased PARP [Poly (ADP-ribose) polymerase] cleavage (apoptotic markers) in various cancer cell lines (Fig. 2a-c). Further, pan-caspase inhibitor Z-VADFMK effectively blocked MGL-mediated cell death (Fig. 2d, e). Together, these results indicate that MGL induces apoptosis in cancer cells via activation of apoptotic signaling.

\section{MGL negatively regulates XIAP and suppression of XIAP is important for MGL-mediated cell death}

To elucidate the mechanism(s) involving MGL-mediated apoptosis, we found that the endogenous levels of XIAP were significantly lower in MGL-overexpressing H1299 cells and HeLa cells (Fig. 3a). These cells inherently exhibit undetectable and low levels of endogenous MGL respectively (Fig. 3a). Conversely, MGL knockdown via RNAi elevated the XIAP protein levels (Fig. 3b). These results suggest that suppression of XIAP appears to play a role in MGL-mediated apoptosis. Results presented in Fig. 3c further indicate that while expression of MGLonly induced significant cell death in various human and mouse tumor cell lines, co-expression of XIAP blocked MGL-mediated cell death; cells expressing vector-only and XIAP-only did not show obvious changes in cell viability (Fig. 3c). MGL overexpression also resulted in significant amount of PARP cleavage and activation of caspases-3 and 9 (Fig. 3d); co-expression of XIAP inhibited MGL-mediated activation (reduction) of procaspase-9, but did not significantly affect MGL-mediated pro-caspase-3 activation (Fig. 3d). These results are consistent with previous studies showing that XIAP binds to monomeric capspase-9 (pro-caspase-9) to prevent caspase-9 activation [17]; however, it only interacts with and inhibits the dimeric caspase-3/activated caspase- 3 and not the pro-caspase/monomeric caspase-3 [19, 20]. Together, these results suggest that MGL is a negative regulator of XIAP and suppression of XIAP appears to play an important role in MGL-mediated cell death.

\section{XIAP expression is elevated in MGL-deficient human and mouse tumor tissues}

Figure 4a shows XIAP protein expression status in mouse embryonic fibroblasts (MEFs) developed from mice with varying MGL status, and as is shown, the expression levels of XIAP were higher in MGL-deficient $\mathrm{MGL}^{+/-}, \mathrm{MGL}^{-/-}$) MEFs (lanes 3-6) compared with MGL-proficient $\left(\mathrm{MGL}^{+/+}\right.$) MEFs (lanes 1 and 2). XIAP expression levels were also generally higher in liver tissues of 5/6 MGL-deficient mice (Fig. 4b, lanes 4-9) compared with those of MGL-proficient mice (Fig. 4b, lanes 1-3). We have shown that significant portion of MGL-deficient $\left(\mathrm{MGL}^{+/-}\right.$and $\mathrm{MGL}^{-/-}$) animals developed lung adenocarcinoma and some developed liver tumors or lymphomas [2]. Figure 4c shows lung tissue of a MGL-proficient $\left(\mathrm{MGL}^{+/+}\right.$) mouse with no tumor (panel a) and lung tissue of a MGL-deficient $\left(\mathrm{MGL}^{+/-}\right)$mouse with lung tumor (panel b). Figure 4c, c and d panels also show microscopic images of a papillary predominant lung adenocarcinoma developed in a MGL-deficient mouse (\#171). Figure 4d shows XIAP protein expression in two groups of mouse lung tissues with different MGL status. As is shown, XIAP expression was significantly higher in lung tissues from MGL-deficient mice relative to tissues from MGL-proficient animals (compare lanes 2-5 and 7-10 with lanes 1 and 6). Protein expression of XIAP in lung tumor tissues of MGLdeficient mouse \#171 (shown in Fig. 4c, panels b, c) was also significantly elevated (Fig. 4d, red arrows). Likewise, lymphoma developed in the liver tissues of MGLdeficient mouse (\#354) (Fig. 4e) exhibited much higher XIAP expression (Fig. 4f, right panel) compared with normal liver tissue of MGL-proficient mouse (\#24) (Fig. 4f, left panel). Thus, it appears that elevated XIAP expression is a common feature of MGL-deficient tissues and may play an important role in tumor development in MGL-deficient animals. We also investigated the possible inverse correlation between MGL and XIAP expression in human lung tissues and noted that MGL 
Fig. 1 Overexpression of MGL induces cancer cell death and activates apoptotic signaling. a, b Overexpression of MGLinduced cell death in three different tumor cell lines. Fortyeight hours following transfection of empty vector or MGL expression construct, representative photomicrographs were taken (a) and cell death was evaluated by trypan-blue exclusion assay (b). Each bar indicates the mean values of three independent experiments $( \pm$ SE, $* p<0.05)(\mathbf{b}) . \mathbf{c}, \mathbf{d}$ Indicated cancer cells were transiently transfected with pDsRedN1-only (RFP, vector) or RFP-MGL (red) for $48 \mathrm{~h}$ followed by DAPI staining (blue) (scale bar $10 \mu \mathrm{m})(\mathbf{c})$. RFP-positive dead cells with apoptotic nuclei (fragment or condensed nuclei indicated by yellow arrows) were evaluated under a fluorescent microscope (d). Each bar indicates the mean values of three independent experiments $( \pm \mathrm{SE}, * p<0.05)$.
A
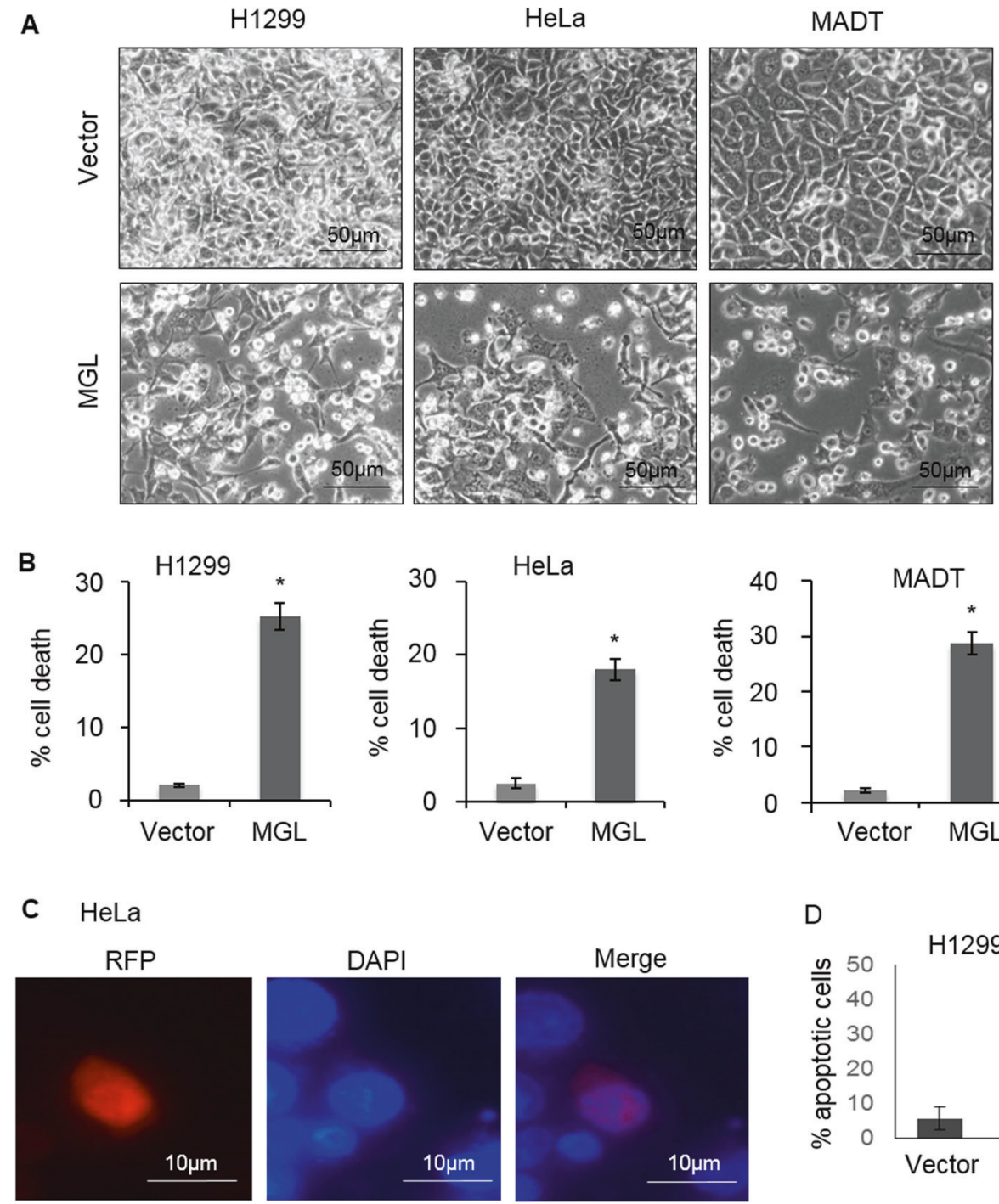

RFP-MGL
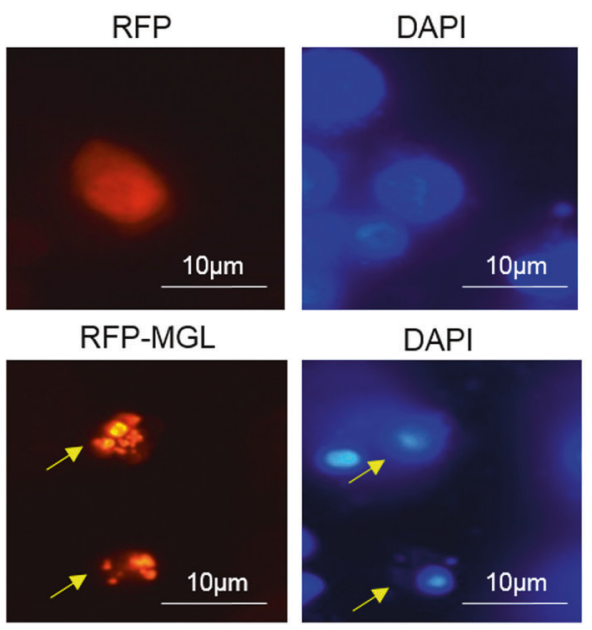

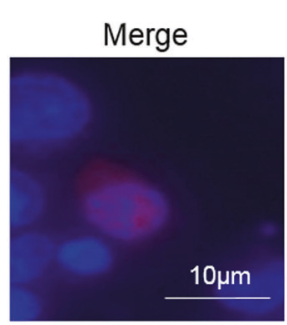

Merge

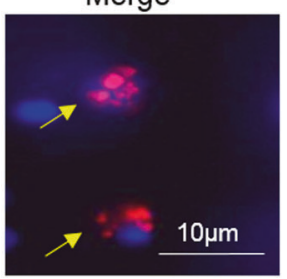

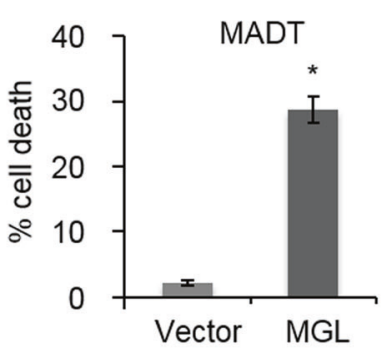

D
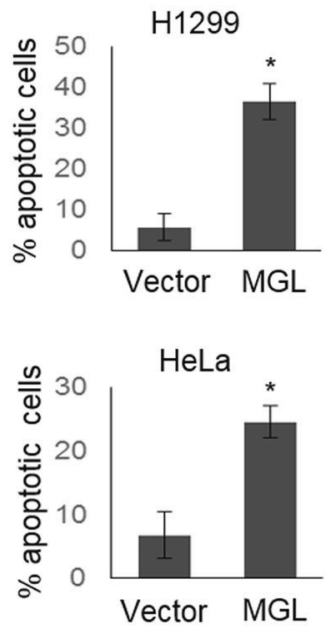

expression was significantly lower in tumor tissues relative to matched normal tissues (Fig. $4 \mathrm{~g}$, upper left and right; Supplementary Fig. 4). In 4/5 lung cancer tissues exhibiting lower MGL levels (red stars), XIAP expression was elevated (Fig. 4g, upper left). Figure $4 \mathrm{~g}$ (lower panel) shows that 9/12 patient lung adenocarcinoma tissues showed a negative correlation between MGL and XIAP. Together, our results indicate a negative correlation between MGL and XIAP in vivo. Accordingly, XIAP induction could play an important role in tumorigenesis under MGL-deficient conditions.

\section{MGL alters XIAP protein stability via proteasomal pathway}

Studies have shown that XIAP is regulated at the protein level by proteasomal pathway [21]. We determined whether MGL alters XIAP protein stability (protein half-life) in MEFs with varying MGL status. Figure 5a, b shows that XIAP protein half-life was much longer in MGL-deficient $\left(\mathrm{MGL}^{+/-}\right.$and $\mathrm{MGL}^{-1-}$ ) MEFs than that in MGL-proficient MEFs. In addition, XIAP protein reduction mediated by MGL was reversed by proteasome inhibitor MG132 in both 293 T cells 

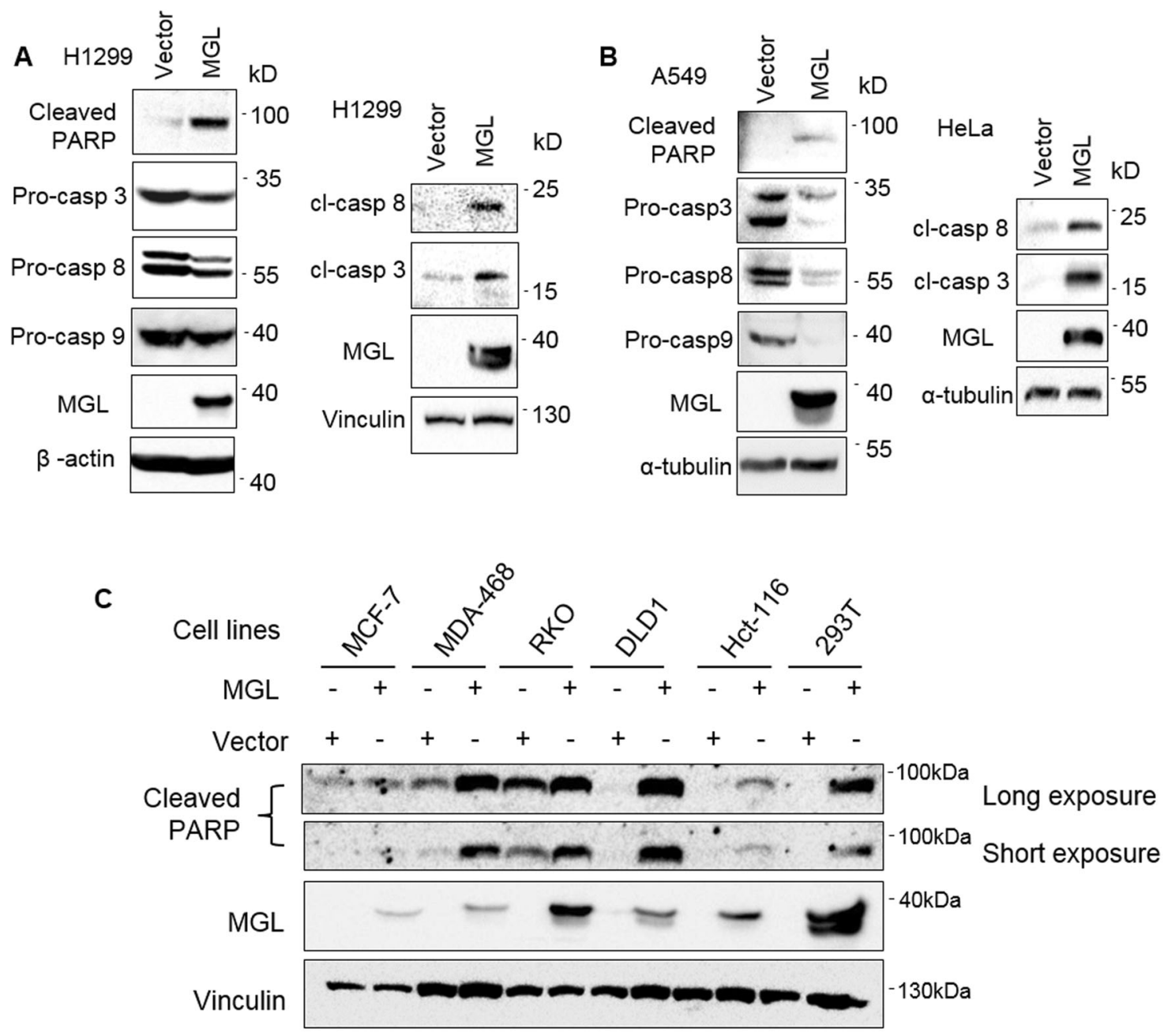

D
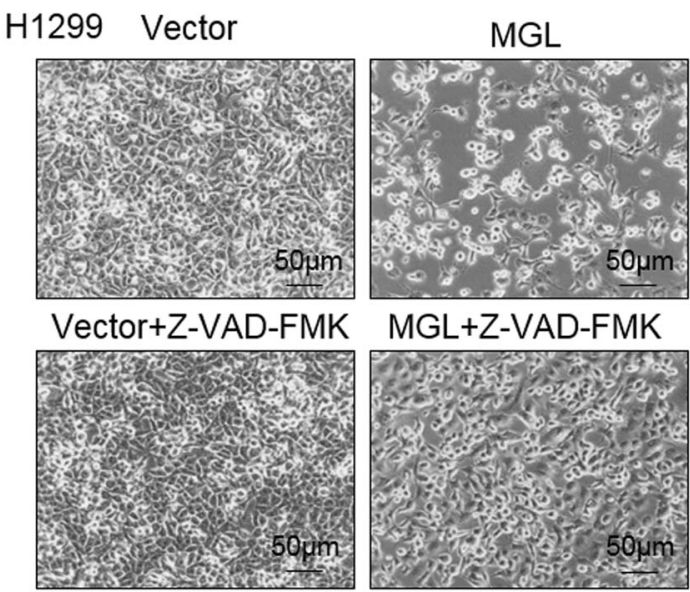

E

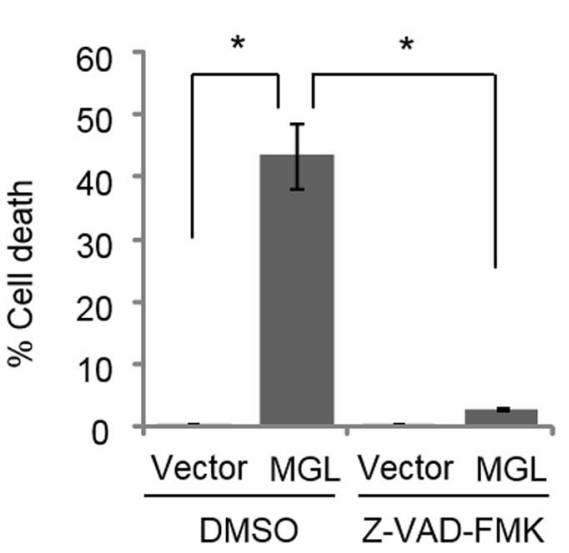

Fig. 2 MGL induces cell death via activation of apoptotic pathways. MGL overexpression triggered PARP cleavage $(\mathbf{a}-\mathbf{c})$ and activation of caspases 8,9 , and 3 , demonstrated by reduction of procaspases (pro-casp) and detection of cleaved caspases (cl-casp) (a, b) in various cancer cell lines. d, e Co-treatment with pan-caspase inhibitor (Z-VAD-FMK, $10 \mu \mathrm{M})$ in $\mathrm{H} 1299$ cells prevented cell death mediated by MGL overexpression ( $48 \mathrm{~h}$ ). Photos were taken $48 \mathrm{~h}$ post transfection; percent of cell death was evaluated as described in legend to Fig. 1. Each bar indicates the mean values of three independent experiments $( \pm \mathrm{SE}, * p<0.01)$. 
A

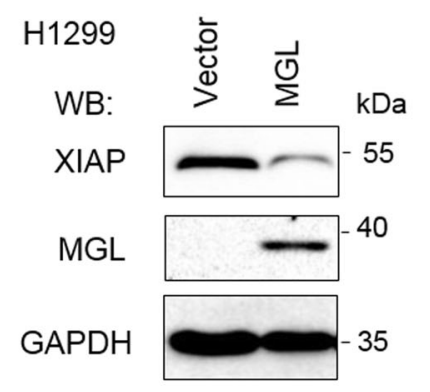

B

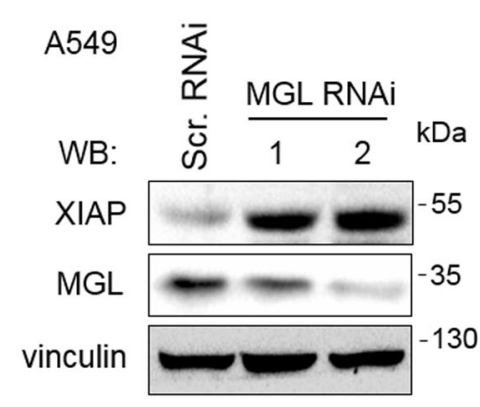

C

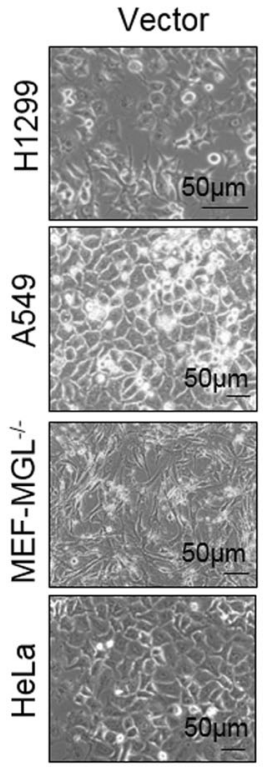

1
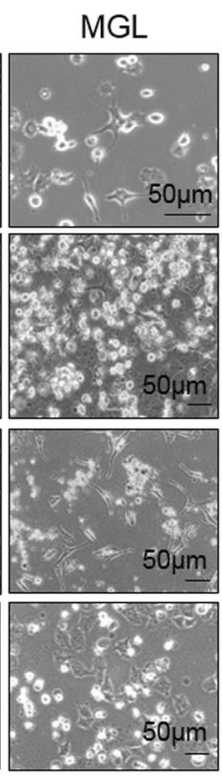

2

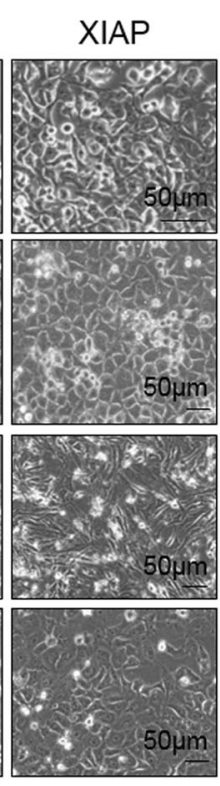

3
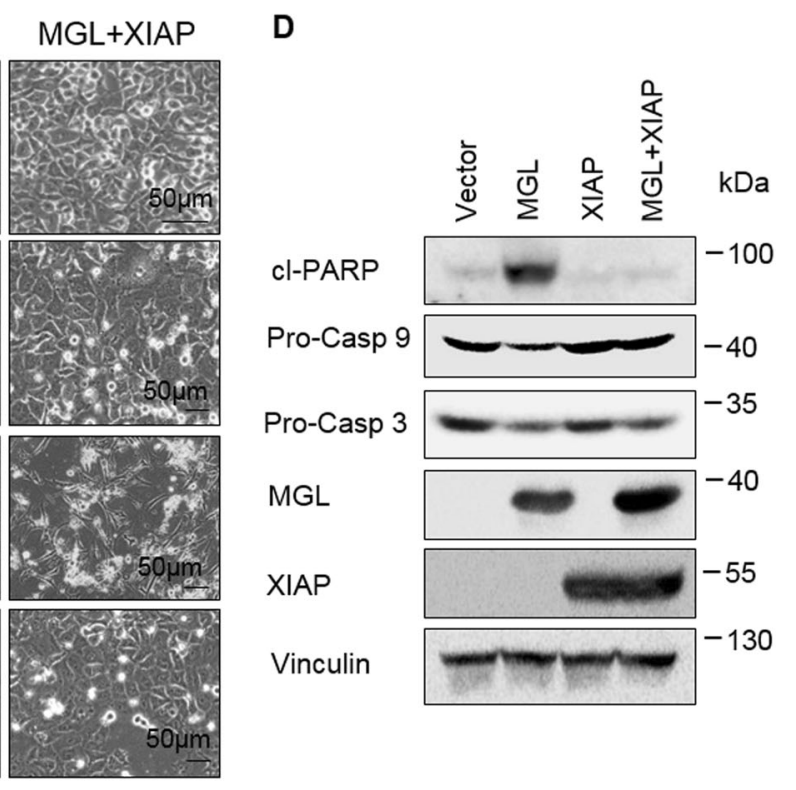
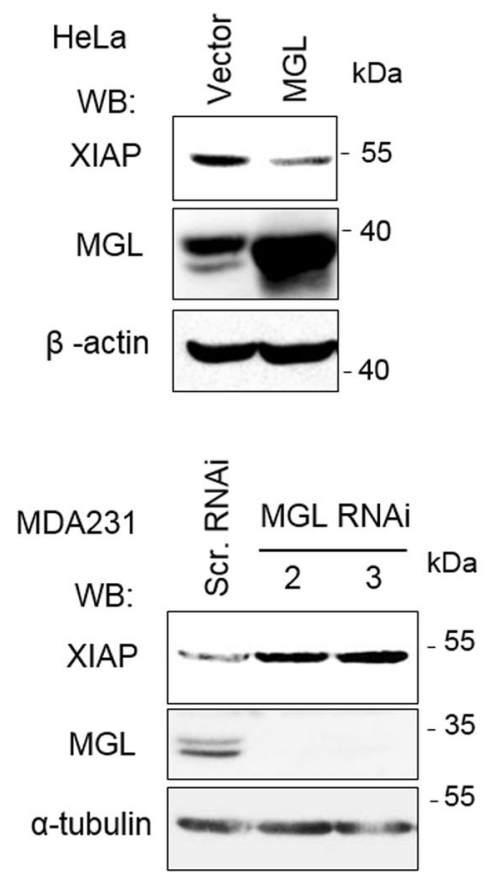

Fig. 3 XIAP suppression is important for MGL-mediated cell death. a Exogenously expressed MGL reduced XIAP (endogenous) protein levels in cancer cells. b XIAP protein levels were significantly elevated in MGL shRNA (MGL RNAi) expressing cells compared with that of scramble RNA (Scr. RNA) expressing cells. $\mathbf{c}$ Exogenous XIAP reversed cell death induced by MGL. Indicated four cell lines transiently expressed either two control vectors (pEBB and $\mathrm{pSR} \alpha-\mathrm{HA}-$

(Fig. 5c) and HeLa cells (Supplementary Fig. 2). Further, XIAP protein ubiquitination was also significantly enhanced in cells overexpressing MGL with (5D) or without
S) (panel 1), or MGL-only (panel 2) or XIAP-only (panel 3) with control vectors, or combined MGL and XIAP (panel 4) vectors; photomicrographs were taken $48 \mathrm{~h}$ post transfection. d PARP cleavage and caspase activation induced by MGL overexpression were suppressed by elevated levels of XIAP. Cell lysates for western analyses were collected $48 \mathrm{~h}$ post transfection.

exogenously expressed ubiquitin (5E). Together, these results suggest that MGL reduces XIAP protein levels by altering its stability via proteasomal pathway. 
A

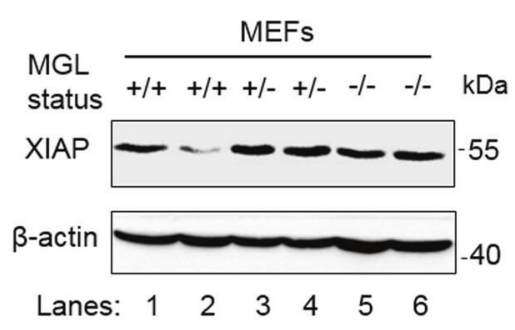

C

\#24 $\mathrm{MGL}^{+/+} \quad$ \#171 $\mathrm{MGL}^{+/-}$lung Normal lung tissue adenocarcinoma

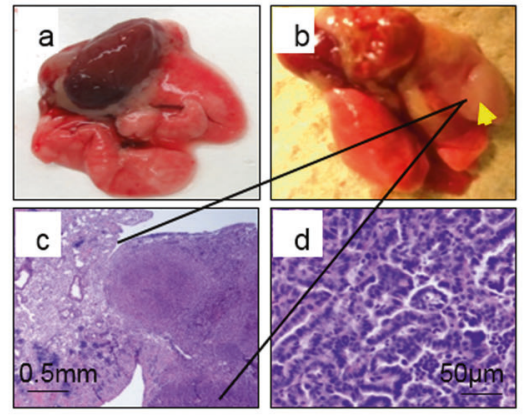

E

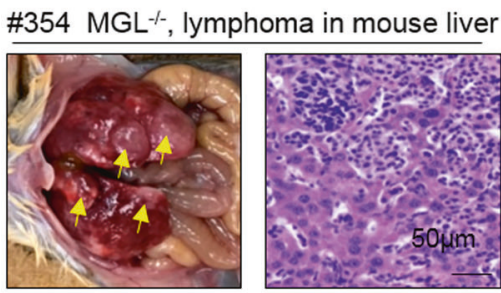

B

MGL

status
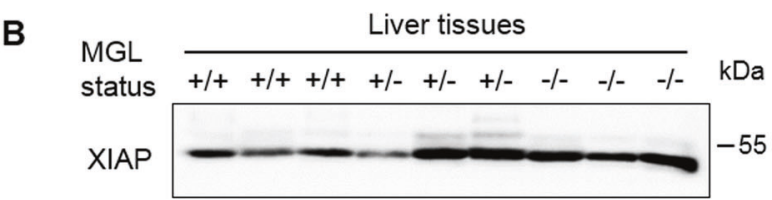

Ponceau S staining

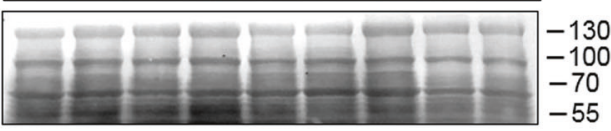

$\begin{array}{llllllllll}\text { Lanes: } & 1 & 2 & 3 & 4 & 5 & 6 & 7 & 8 & 9\end{array}$

D Mouse Lung tissues

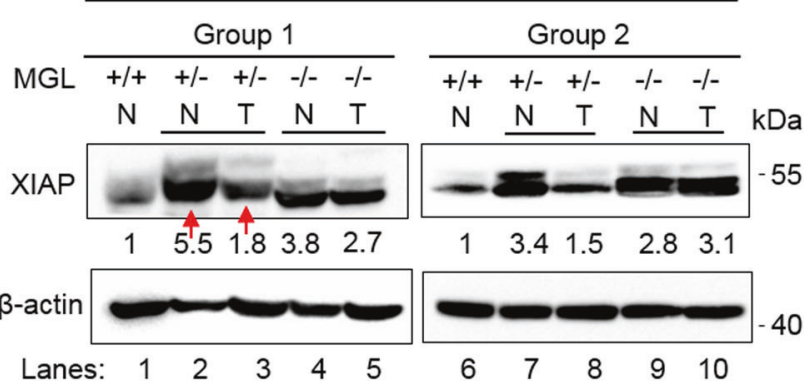

F Mouse tissue IHC staining with XIAP antibody \#24 $\mathrm{MGL}^{+/+} \quad$ \#354 $\mathrm{MGL}^{-/-}$

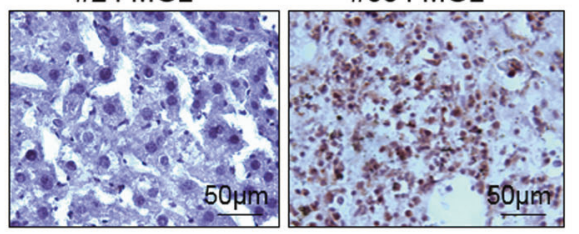

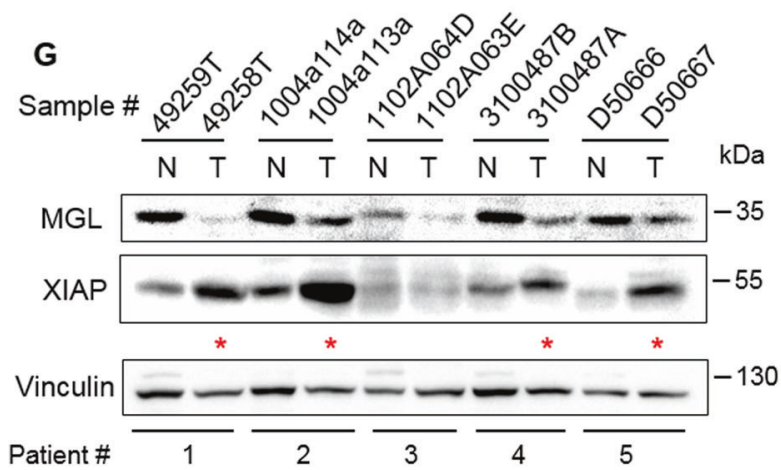

MGL Immunohistochemistry staining Patient sample 3091427A (lung)

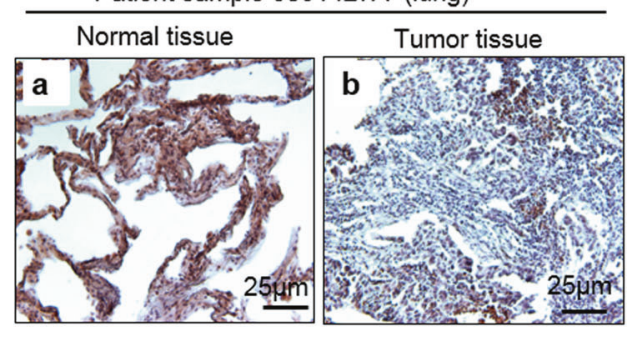

\begin{tabular}{lccc}
\multicolumn{4}{c}{ XIAP-MGL expression in matched human lung normal \& cancer samples } \\
\hline $\begin{array}{l}\text { Patient sample } \\
\text { studied }\end{array}$ & $\begin{array}{c}\text { MGL reduced } \\
\text { in tumor }\end{array}$ & $\begin{array}{c}\text { XIAP increased } \\
\text { in tumor }\end{array}$ & $\begin{array}{c}\text { Negative correlation btw. } \\
\text { MGL \& XIAP * }\end{array}$ \\
\hline 12 & $10(83 \%)$ & $9(75 \%)$ & $9(75 \%)$
\end{tabular}

* As shown in the left (samples marked by red star).

\section{MGL directly interacts with XIAP via its $\mathbf{N}$-terminus}

We also investigated whether MGL interacts with XIAP. Figure 6a shows that the S-tagged MGL, but not the S-tagalone, pulled down endogenous XIAP in H1299 and HeLa cells. In addition, endogenous MGL and XIAP were co- precipitated by XIAP antibodies not by control $\operatorname{IgG}$ (Fig. 6b). These results suggest that MGL interacts with XIAP. To determine the nature of MGL-XIAP interaction, in vitro protein interaction assays were performed using the purified recombinant XIAP and MGL proteins. As seen in Fig. 6c, recombinant S-MGL, but not recombinant 
Fig. 4 Expression of XIAP is elevated in MGL-deficient human and animal tissues. a, $\mathbf{b}$ XIAP protein expression in MEFs and liver tissues extracted from MGL-proficient $(+/+)$ or -deficient $(+/-$ or $-/-$ ) mice. $\beta$-actin or Ponceau S staining was used as protein loading controls. c (a) Lung tissue of $\mathrm{MGL}^{+/+}$mouse; (b) non-tumor and tumor (yellow arrow) lung tissues of $\mathrm{MGL}^{+/-}$mouse; (c, d) H\&E staining of $\mathrm{MGL}^{+/-}$lung tumor tissues [shown in (b)] imaged under microscope at $4 \times$ (c) or $40 \times$ (d) magnification. d XIAP expression is elevated in MGL-deficient lung tissues. Lysates of lung tissue used in lanes 2 and 3 (red arrows) were from the same animal (\#171) shown in c, b panel. e H\&E staining (right) of lymphoma tissues grown in $\mathrm{MGL}^{-1-}$ animal (\#354) liver tissue (left, yellow arrows). f XIAP immunostaining of mouse liver tissues. Left: normal liver tissue $\left(\mathrm{MGL}^{+/+}\right)$. Right: lymphoma grown in mouse liver (\#354). $\mathbf{g}$ Expression patterns of XIAP and MGL in matched human lung normal and tumor tissues. Left: representative results of western blot analyses showing negative correlation between MGL and XIAP protein expression (denoted by *). Right: MGL immunostaining of human lung normal (a) and tumor tissues (b). Bottom: summary of human lung cancer tissue studies.

S-protein-only, pulled down purified recombinant XIAP demonstrating that MGL directly interacts with XIAP. To study the region(s) of MGL involved in XIAP interaction, we generated a set of MGL-deletion variants (Fig. 6d and legend to Fig. 6d). The expression of MGL full-length and various deletion variants are shown in Fig. 6e, f (inputs, bands indicated by asterisk $(*))$. Our results indicate that XIAP was abundantly co-precipitated with variant $1-105$ and full-length MGL (Fig. 6e, lanes 6, 14, 8, and 16) but not with MGL variant harboring residues 106-313 (Fig. 6e, lane 7) in HeLa cells. These results indicate that the XIAPinteraction region on MGL resides at the N-terminus. Interestingly, although the MGL variant 1-209 also harbored the N-terminus, it showed weaker interaction with XIAP in both HeLa and H1299 cells (Fig. 6e, lane 15 and Fig. 6f, lane 7). Thus, these results show that (i) MGL central region (a.a. 106-209) does not mediate MGL-XIAP interaction, (ii) the existence of the central region (a.a. 106-209) may impede the $\mathrm{N}$-terminal region to interact with XIAP, acting through a possible intramolecular negative regulatory mechanism $[22,23]$.

\section{XIAP interacts with MGL via its C-terminus}

We also sought to identify the MGL-interacting region of XIAP. We used various XIAP deletion variants that lacked the N-terminal, central, and C-terminal regions of the protein [24] as shown in Fig. 7a. Expression of the full-length and deletion variants of XIAP was determined as shown in Fig. 7b, c, (inputs lower panels, bands indicated by asterisk $(*))$. As we show in Fig. 7, S-tagged MGL-1-105 or FLMGL abundantly co-precipitated with full-length XIAP protein as well as XIAP variant devoid of three BIR domains ( $\triangle \mathrm{BIR}$, a.a. 331-497) in both $293 \mathrm{~T}$ and HeLa cells (Fig. 7b, c, top panels). Consistent with this finding, MGL did not interact with XIAP deletion variant containing only the BIR domain (BIR1-2-3, a.a. 1-350) (Fig. 7b, c, top panels). These results indicated that BIR domains of XIAP may not interact with MGL. In comparison with MGL interaction with full-length XIAP and the variant containing UBA and RING domains ( $\triangle \mathrm{BIR}$ ), the RING deletion variant ( $\triangle$ RING, 1-449) had significantly less interaction with MGL, however, the interaction was not completely abolished (Fig. 7b, c). Collectively, these results indicate that RING domain of XIAP is important for MGL interaction and the UBA region may also be partly involved in this interaction.

\section{$\mathrm{N}$-terminal region of MGL is sufficient to kill cancer cells}

Next, we sought to determine the region of MGL that is involved in cancer cell killing. As shown in Fig. 8a, variant 1-105 and full-length MGL induced cell death in both HeLa and H1299 cancer cells whereas variant 1-209 did not. In Fig. 6, we show that variant 1-105 and FL-MGL interacted with XIAP while MGL-1-209 did not. Thus, the cell killing potential of MGL appears to be associated with its ability to interact with XIAP. Cells expressing increased amount of MGL-1-105 or FL-MGL exhibited lesser survival (Fig. 8a, right panel) suggesting that MGL N-terminal region-alone is sufficient to kill cancer cells. Figure $8 b$, c and Supplementary Fig. 3 further demonstrate that MGL1-105 and FL-MGL, but not 1-209 and other variants, were able to effectively induce cleavage (activation) of caspase-3 (cl-casp3) and PARP (cl-PARP). Further experiments showed that expression of MGL first 60 a.a. (1-60) was sufficient to induce cleavage (activation) of caspase- 3 and PARP (Fig. 8c). As shown in Fig. 8d, deletion of MGL gene significantly enhanced cell survival in MGL-deficient MEFs under anticancer drug (doxorubicin, etoposide, and taxol) treatments $(p<0.05)$. These results indicate that MGL plays an important role in regulation of cell death and the death inducing region of MGL resides at its $\mathrm{N}$-terminus. In light of these results, we propose that the N-terminal region of MGL could have a potential to be developed as an anticancer therapeutic.

\section{Discussion}

In this study, we have identified a novel cellular function of MGL in that it works as a negative regulator of XIAP. XIAP is one of the major inhibitors of apoptosis and known to inhibit caspases-3, 7, and 9 [25]. Overexpression of MGL activated caspases, and induced PARP cleavage and apoptosis (Figs. 1-3). We show that lipase dead-mutant of MGL was able to also induce cell death and activate caspases in a manner similar to that induced by the wild-type counterpart 
A
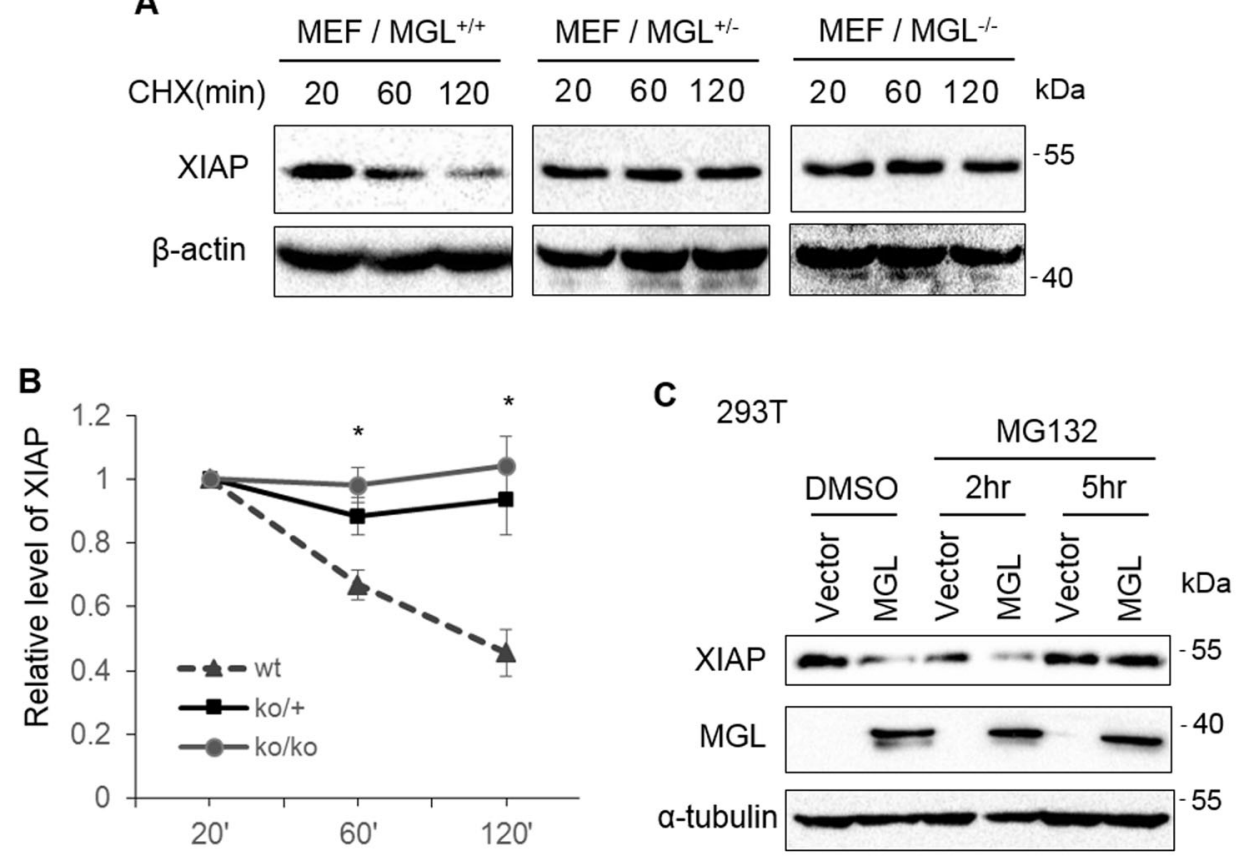
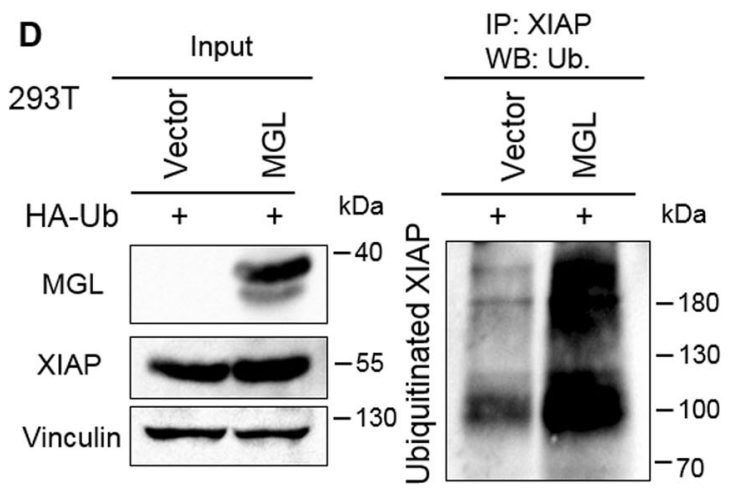

Fig. 5 MGL alters XIAP protein stability via proteasomal pathway. a XIAP protein stability is increased in MGL-deficient MEFs. Protein synthesis was first blocked by treatment of cycloheximide (CHX) for indicated times and reduction of XIAP (protein decay) was then measured by western blot analyses. b Quantitative results of XIAP protein level in MEFs with different MGL status shown in a. Relative band intensity of XIAP (a, upper panels) was adjusted to the band intensity of the loading control $\beta$-actin (a, lower panels). The first time points $(20 \mathrm{~min})$ in each cell type were set as 1 . Each bar indicates the mean values of three independent experiments $( \pm \mathrm{SE} * p<0.05)$. c XIAP protein reduction is reversed in MGL-overexpressing cells

(Supplementary Fig. 5a-c). Furthermore, treatment with two different MGL inhibitors URB602 and JZL184 did not alter MGL-mediated growth inhibition (Supplementary Fig. 6). MGL inhibitor JZL184 also did not inhibit cell growth when used at $10 \mathrm{nM}$ or $1 \mu \mathrm{M}$ in several cell lines. These concentrations are higher than IC50 value at $8 \mathrm{nM}$ of JZL184 [26]. JZL184 inhibited cell growth at $10 \mu \mathrm{M}$, a concentration many fold higher than its IC50 value (Supplementary Fig. 7). Given that JZL184, at higher
E

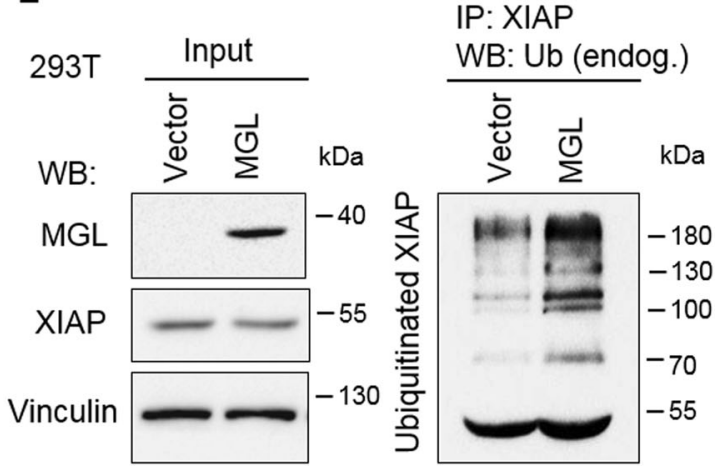

following treatment with proteasome inhibitor MG132. Forty-eight hours following transfection of indicated vectors, cells were harvested after treatment of MG132 for indicated times. Untreated (DMSO) samples were also harvested at the 5-h time point. d Overexpression of MGL increases XIAP ubiquitination levels. Vector-only or MGL vector was co-transfected with ubiquitin vector (HA-Ub) for $48 \mathrm{~h}$, then the cells were treated with MG132 for $6 \mathrm{~h}$. Immunoprecipitation (IP) was performed using ubiquitin antibody. e MGL promotes XIAP ubiquitination by endogenous ubiquitin. Cells were transfected with vector-only or MGL expression vector for $48 \mathrm{~h}$, then treated with MG132 for $6 \mathrm{~h}$ prior to harvesting.

concentrations, reported to also inhibit fatty acid amide hydrolase [26], JZL184-mediated growth inhibition at higher concentration may involve effect on fatty acid amide hydrolase. Taken together, although MGL is a lipase, MGLmediated apoptosis appears to be independent of its lipase activity.

Our results show that MGL can regulate cellular functions through protein-protein interactions. We show that overexpression of MGL significantly reduced XIAP levels; 

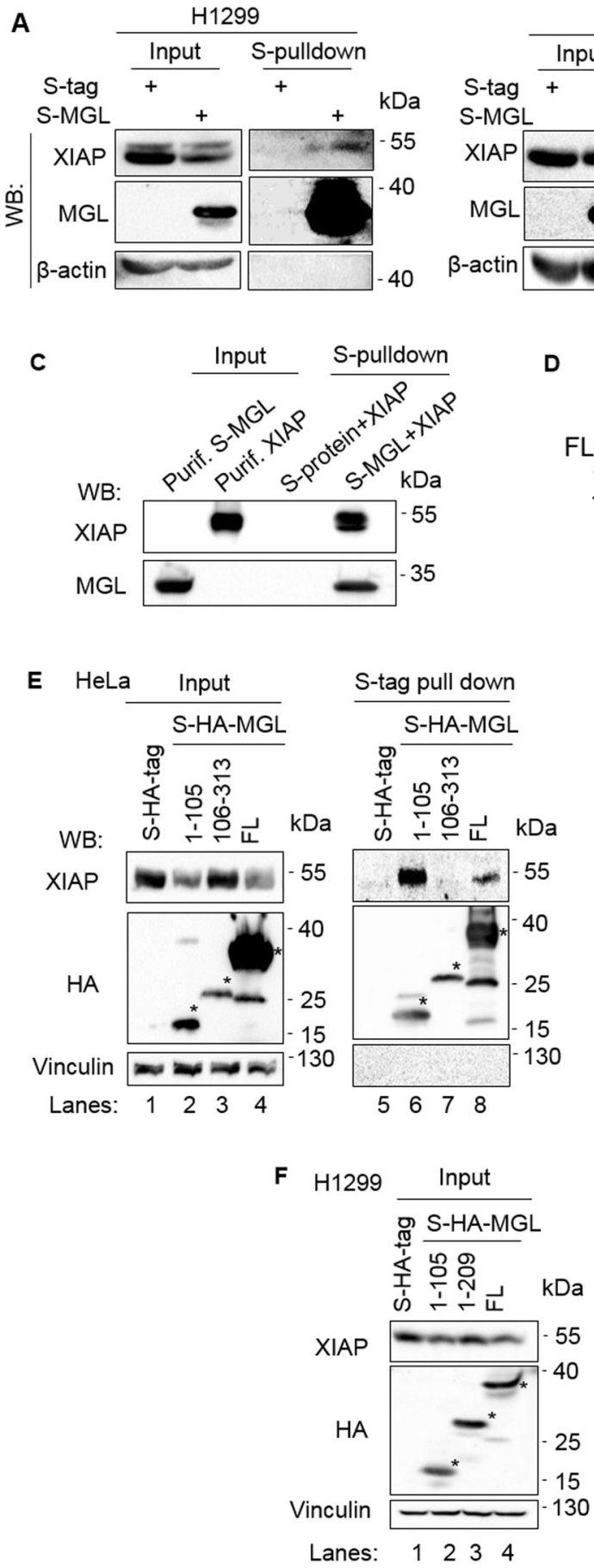

Fig. 6 MGL directly interacts with XIAP. a Exogenous S-tagged MGL, but not S-tag-only, pulls down endogenous XIAP. Cells were transfected with indicated vectors for $48 \mathrm{~h}$ prior to harvesting. S-tag pulldown experiments were performed as described in "Materials and methods". b Endogenous interactions between MGL and XIAP demonstrated by co-immunoprecipitation. Cells were treated with MGL132 for $6 \mathrm{~h}$ prior to harvesting. Immunoprecipitation (IP) was performed using control IgG or XIAP antibody. c Recombinant XIAP directly interacts with purified recombinant MGL in vitro. d A schematic illustration of full-length and deletion variants of MGL. The numbers indicate the amino acid positions of the full-length MGL. MGL-deletion variants include the N-terminus-only (1-105); N-

\section{B}

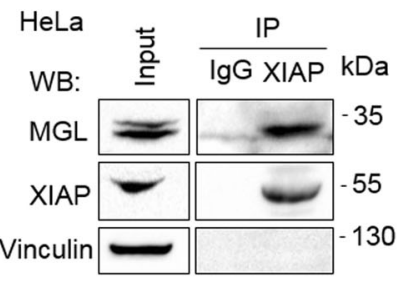

D

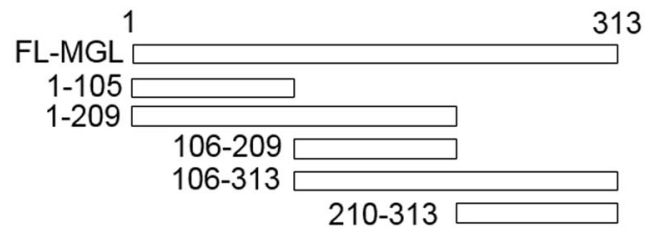

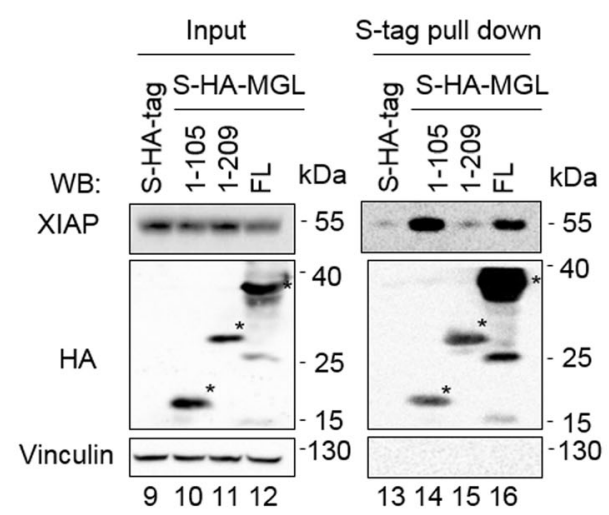

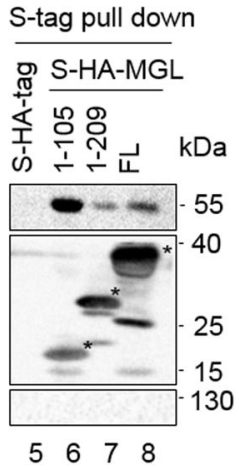

terminus plus the central region (1-209); central region-only (106-209); central region plus C-terminus (106-313, which contains the entire MGL lipase catalytic triad) and the region of C-terminusonly (210-313). The correct nucleotide sequences of all MGL-deletion variants were confirmed by DNA sequencing. e, f Interaction of endogenous XIAP with exogenously expressed MGL-1-105 and fulllength MGL. Lysates from cells (HeLa in e, H1299 in f) expressing (48 h) S-HA-tagged full-length (FL)-MGL or S-HA-tagged MGLdeletion variants or S-HA-tag-only were assayed for western blotting (inputs) or proceeded for S-tag pulldown assays. The S-tag-pulldown products were assayed for protein interactions using XIAP antibody. The correct-sized MGL protein products are marked with asterisk “*”. 
A XIAP constructs

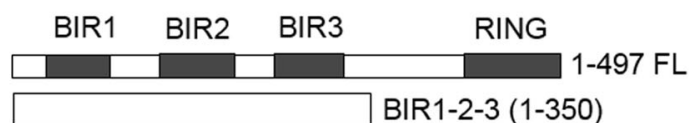

$\Delta R I N G(1-449)$

$\Delta \mathrm{BIR}(331-497)$

B

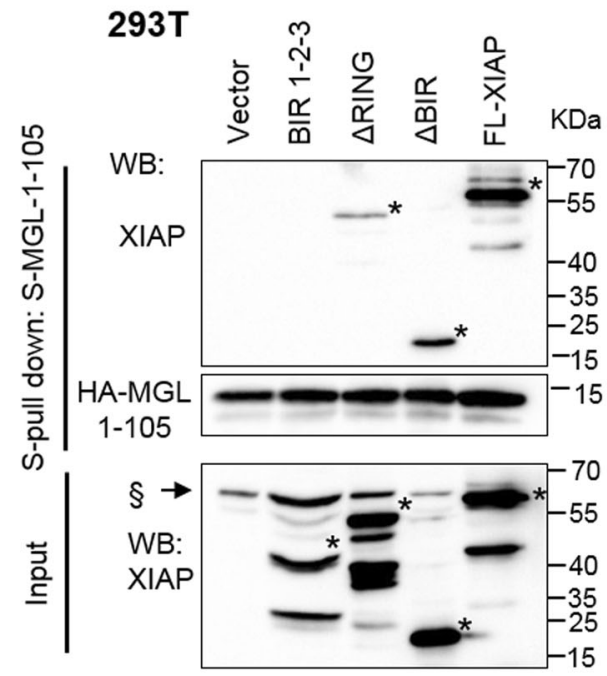

C

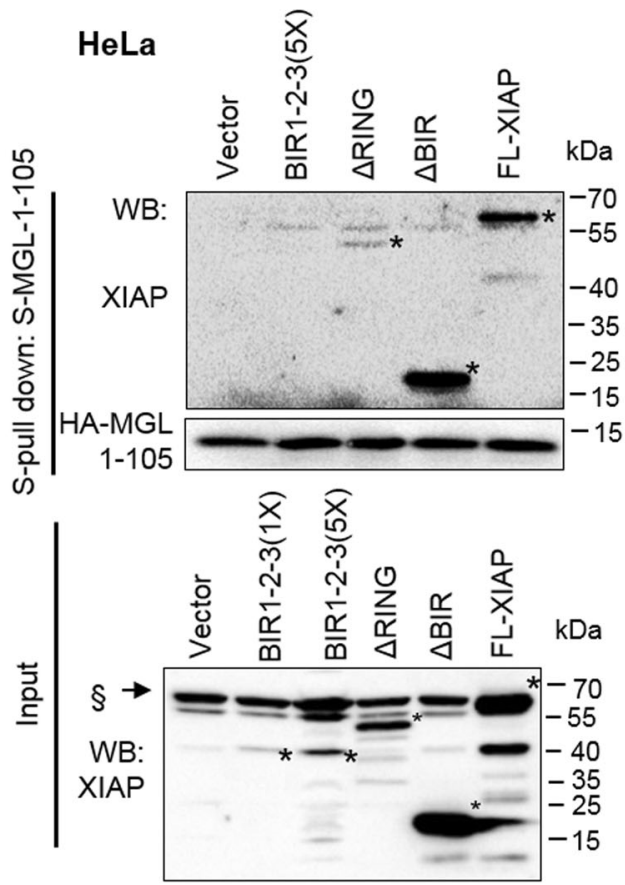

Fig. 7 XIAP interacts with MGL via its C-terminus. a A schematic illustration of full-length and deletion variants of XIAP. The numbers indicate the amino acid positions of the full-length XIAP. b, c Stagged MGL-1-105 (left panels in $\mathbf{b}$ and $\mathbf{c}$ ) or FL-MGL (right panels in $\mathbf{b}$ and $\mathbf{c}$ ) was used in the S-tag pulldown down experiments. Protein inputs are shown in the bottom panels; "*” sign indicates the correctsize of exogenous XIAP variants. Bands for endogenous XIAP protein is indicated with the sign "§". For S-tag pulldown experiments, cell
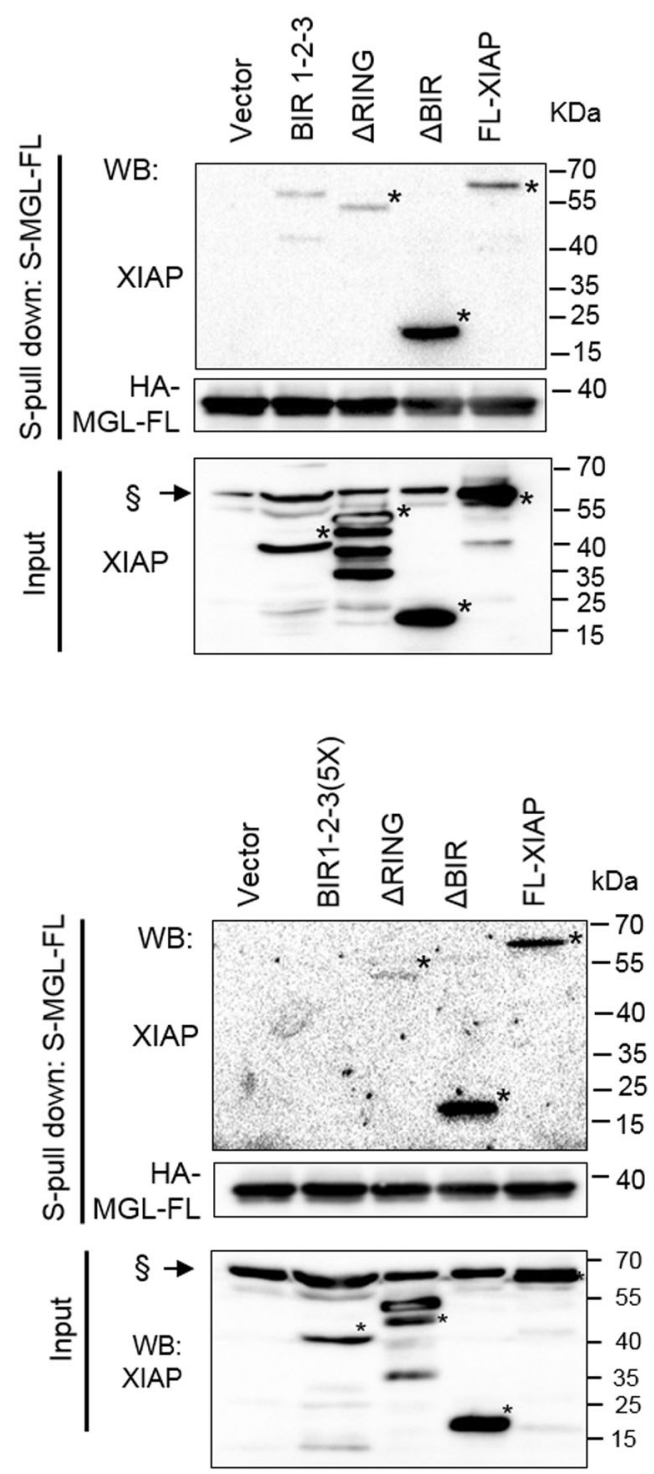

lysates expressing S-MGL-1-105 or S-MGL full-length (FL) were mixed separately with cell lysates expressing full-length-XIAP (FLXIAP) or deletion variants or control vector. In HeLa cells, the expression of BIR 1-2-3 was relatively low (Fig. 8c, left lower panel, $1 \times)$; therefore, cell lysates with BIR 1-2-3 expressions were increased five times [BIR 1-2-3 (5x)] as much in the protein pulldown experiments (c, top panels). 
A

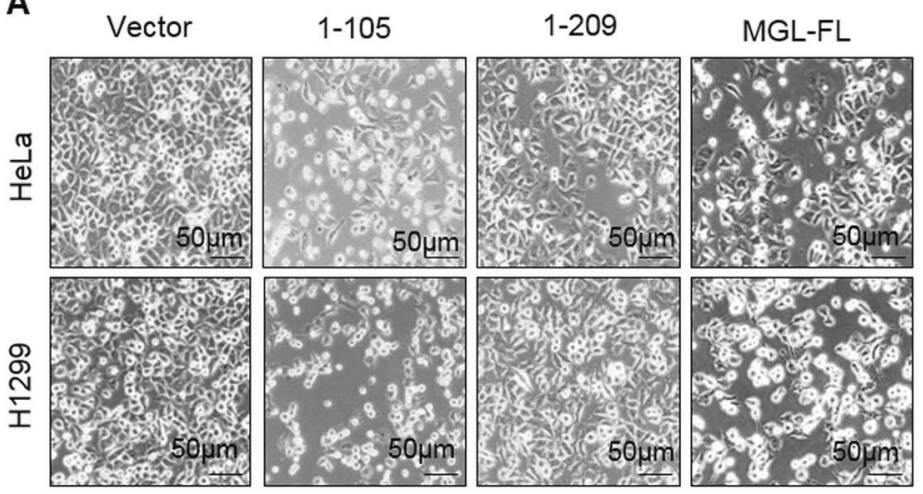

HeLa MTT

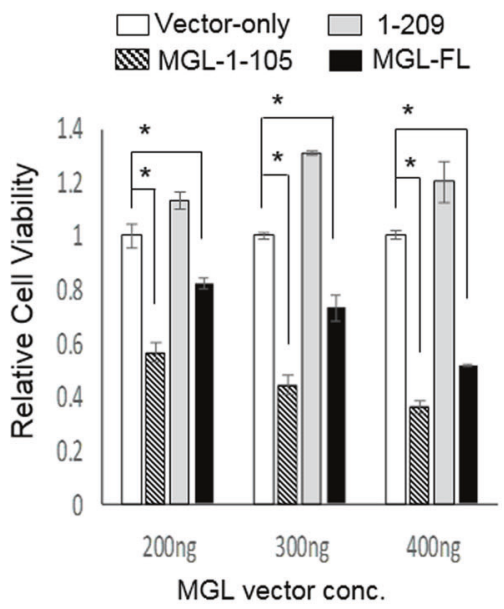

C

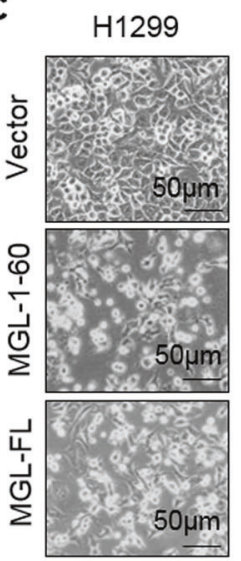

D

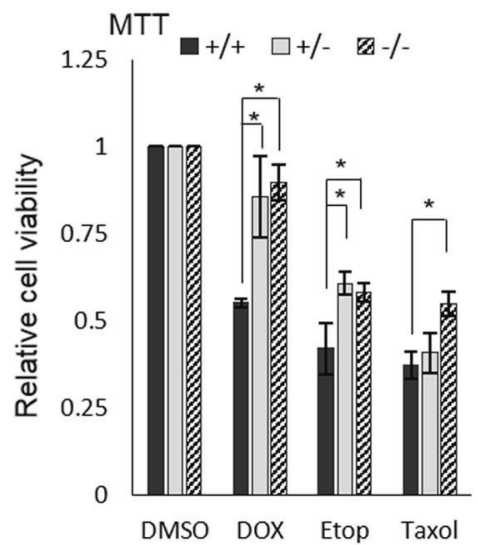

Fig. 8 N-terminus of MGL is critical for MGL-mediated cancer cell killing. a N-terminal domain of MGL induces apoptosis. Cells, transfected with indicated vectors for $48 \mathrm{~h}$, were imaged under a microscope (left) and harvested for MTT assays (right). Each bar indicates the mean values of three independent experiments $( \pm \mathrm{SE}$, $* p<0.05)$. b Expression of MGL N-terminus domain $(1-105)$ and FL-MGL induces caspase-3 cleavage. HeLa cells were transfected with indicated MGL variants for $48 \mathrm{~h}$ prior to harvesting. Cl-casp3; cleaved caspase-3. HA antibody detects all HA-tagged MGL variants. c MGL fragment containing first 60 amino acids is sufficient to induce

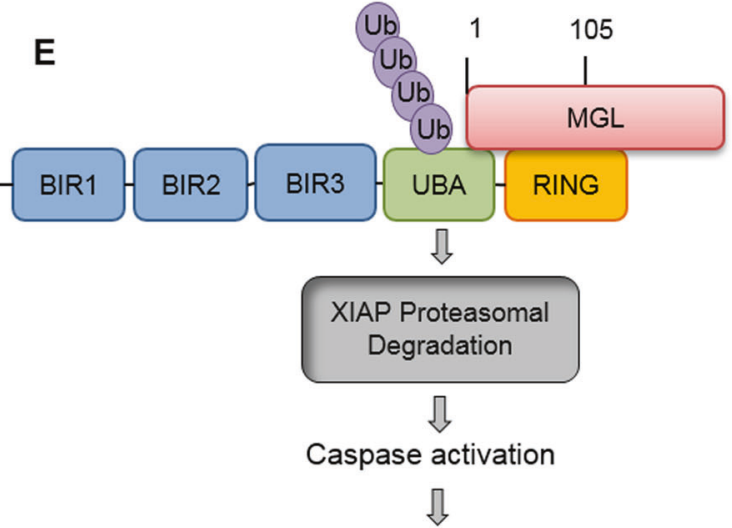

Cell death

cell death in H1299 cells. Photomicrographs were taken for cells transfected $(48 \mathrm{~h})$ with indicated vectors (left) and cell lysates were harvested for western blot analysis (right). d MGL-deficient cells are more resistant to treatment by chemotherapeutic drugs. MTT assays were performed in MGL-proficient $(+/+)$ or -deficient $(+/-,-/-)$ MEFs following the treatments ( $48 \mathrm{~h}$ ) with doxorubicin (DOX, $40 \mathrm{ng}$ / $\mathrm{ml}$ ), etoposide (Etop, $50 \mu \mathrm{M})$, and taxol $(10 \mu \mathrm{M})$. For bar graphs, each bar indicates the mean values of three independent experiments $( \pm \mathrm{SE}$, ${ }^{*} p<0.05$ ). e A proposed model illustrating the possible mechanism via which MGL induces cell death in cancer cells. 
conversely, depletion of MGL by RNAi significantly enhanced XIAP levels (Fig. 3). Further mechanistic studies demonstrated that MGL altered XIAP protein stability; XIAP protein half-life was significantly longer in MGL-deficient cells (Fig. 5a, b). Furthermore, elevated expression of MGL enhanced XIAP protein ubiquitination (Fig. 5d, e) and treatment with proteasome inhibitor MG132 prevented MGLmediated XIAP protein reduction (Fig. 5c and Supplementary Fig. 2). Treatment with XIAP inhibitor Embelin [27] did not further enhance MGL-mediated growth suppression (Supplementary Fig. 8). Together, these results demonstrate that MGL negatively controls XIAP levels by enhancing its protein ubiquitination and degradation. A schematic illustration of the proposed model depicting MGL involvement in regulation of cell death is presented in Fig. 8e.

A study [28] has previously reported that MGL behaved as an oncogenic protein and promoted tumor metastasis due to its lipase activity. Our current and previous findings indicate that MGL modulates cellular function by regulating various proteins. For example, MGL inhibits expression and phosphorylation of EGFR and Akt/ERK [2], and downregulates COX-2 [2] and XIAP (current study). MGL expression is also reduced in malignancies of many organs including those affecting lung, liver, breast, and several others [1-5]. MGL expression is elevated in some tumors such as kidney cancer [6]. MGL-mediated downregulation of XIAP, and growth suppression, and apoptosis were noted in the majority of cancer cell lines studied here. In two cell lines including OVCAR3 and SKOV3 such effects were not evident (Supplementary Figs. 9, 10). MGL-knockout animals also developed neoplasia in certain tissues such as lung, liver, and lymphatic tissues but not in all tissues [2]. Thus, MGL regulation and function appear to be cell/tissuetype specific particularly in relation to tumorigenesis. It is possible that MGL may have a dual function as a tumor suppressor or oncoprotein as dictated by cell and tissue type. Several proteins, such as Notch, Spleen Tyrosine Kinase, Sirtuins, WT- 1 , and transforming growth factor- $\beta$ were also reported to have such dual functions [29-33].

XIAP is known to form homodimer [34] and is selfubiquitinated (autoubiquitination) involving its own RING domain, which results in protein degradation [21, 35, 36]. MGL does not contain a RING domain and is not expected to be an E3-ligase. MGL may mediate its negative effect on XIAP via one or more mechanisms. For example, MGL may alter the E3-ligase activity of XIAP via direct interaction with XIAP (Fig. 6a-c) resulting in enhancement of XIAP autoubiquitination and degradation. Previous studies have demonstrated that the E3-ligase activity of BRCA1 is significantly enhanced by direct interaction with a protein called BARD1 [37]. BRCA1 directly interacts with BARD1 protein via its RING domain and the direct interaction between these two proteins results in the enhancement of
BRCA1 E3-ligase activity and autoubiquitination [37]. It is also possible that MGL may work to enhance XIAP ubiquitination by facilitating XIAP to form dimers that subsequently promote intermolecular autoubiquitination of two XIAP molecules. Previous studies have shown that human MGL crystallized as a dimer [38] although another study has shown MGL to be a monomer [39]. Our unpublished results indicate that MGL forms homo-oligomers. We found that S-tagged MGL was able to pull down (precipitate) the RFP-tagged MGL in protein lysates of cells co-expressing these two differently tagged MGL constructs. Thus, it is possible that when MGL forms oligomers and interacts with XIAP, it brings two XIAP molecules into close proximity facilitating XIAP's homodimerization, autoubiquitination, and protein degradation.

We have also identified the XIAP region responsible for interaction with MGL. Our results show that deletion of the RING domain ( $\triangle$ RING) significantly reduced XIAP interaction with MGL compared with the full-length counterpart, whereas deletion of BIR domains $(\triangle \mathrm{BIR})$ did not affect XIAP interaction with MGL (Fig. 7b, c). In addition, a fragment containing only the BIR domains (BIR-1-2-3) did not show interaction with MGL (Fig. 7b, c). Thus, our results indicate that the C-terminus of XIAP including the RING domain, rather than the N-terminal BIR domains, interacts with MGL. Previous studies have shown that XIAP N-terminal BIR-2 or BIR-3 domains interact with caspases-3, 7, or 9 [12-15, 17]. Presumably, MGL-XIAP interaction may interfere with XIAP binding to caspases and thus, protect caspases from XIAP-mediated protein degradation and inhibition. Smac/ DIABLO, an inhibitor of XIAP released from mitochondria in response to apoptotic stimuli, promotes apoptosis via interaction with XIAP and averts XIAP interaction with caspases [40]. It will be of great interest in the future to investigate whether MGL possesses Smac/DIABLO-like function to counter inhibition of caspases and enhances activities of caspases via its interaction with XIAP.

We also show that N-terminus of MGL is important for MGL-mediated cell death and interaction with XIAP. We demonstrate that MGL N-terminal fragment (a.a. 1-105) induces cleavage (activation) of caspase- 3 and PARP, and cell death, which is coincident with its interaction with XIAP (Fig. 6). Another MGL fragment (1-209), which contains the N-terminal first 105 residues plus 104 amino acids at the central region, did not induce caspase- 3 activation, PARP cleavage, and cell death (Fig. 8a, b). MGL fragment 1-209 also did not or minimally interacted with XIAP (Fig. 6e, f). We also noted that the N-terminal polypeptide 1-105 killed cells better than the FL-MGL when expressed in cells under similar conditions (Fig. 8a). MGL-1-105 also had stronger interaction with XIAP than FL-MGL (Fig. 6e, f). Based on these results, we propose that the central region (a.a. 106-209) may harbor regulatory 
element(s) that functionally suppresses the action of the Nterminus of MGL on cell killing and interaction with XIAP. When the inhibitory central domain (a.a. 106-209) is removed, the suppression on N-terminus-mediated XIAP interaction and cell killing is alleviated, such that the fragment 1-105 works better than FL-MGL for its XIAP interaction and cell killing. Rotem-Bamberger et al. [23] have previously shown that ASPP2 (Apoptosis Stimulating Protein of p53, also called p53BP-2) stimulates the apoptotic function of p53 via its interaction with p53; the Cterminus of ASSP2 interacts with p53 while the N-terminus acts as intramolecular negative regulatory element to inhibit the p53-ASSP2 interaction. It remains to be determined whether the central region of MGL has a suppressive role on the N-terminus in protein interactions (i.e., with XIAP) and cell killing.

Overexpression of XIAP has been commonly found in human cancers [41-44] and the mechanism(s) for its elevated expression are not very clear. Our studies indicate that MGL deficiency could be one of the important mechanisms leading to XIAP overexpression. We show that XIAP expression is significantly lower in MGL-overexpressing cells and opposite is true in MGL-deficient cells (Fig. 3). Such reciprocal expression patterns of these two proteins were also noted in MGL-knockout animal tissues and MEFs as well as in human lung cancer tissues (Fig. 4). MGL expression is commonly reduced in many human malignancies $[1-3,5,6]$. Studies presented here, for the first time, provide evidence suggesting a possible causative link between MGL deficiency and XIAP overexpression.

Our results also indicate that MGL-deficient cells are more resistant to doxorubicin and etoposide, the anticancer drugs commonly used in the clinic (Fig. 8d). These results suggest that reduced or lack of MGL expression may contribute to anticancer drug resistance. Our previous studies have also demonstrated that lack of MGL expression resulted in elevated Akt phosphorylation [1, 2]. Thus, our current and previous studies together demonstrate that MGL appears to regulate cell death and thus, its tumorsuppressive function via at least two mechanisms: one is to inhibit the PI3K/Akt survival pathway and another is to block the function of XIAP by enhancing its protein degradation. Currently, several clinical trials are under way using SMAC-mimetic LCL161 in combination with other anticancer agents for treatments of relapsed and/or refractory small cell lung cancer, gynecologic malignancies, and multiple myeloma [45]. Our study has revealed that a small region (a.a. 1-60) on the N-terminus of MGL (MGL-N) has a strong pro-apoptotic potential (Fig. 8c). It will be of great interest to further determine whether MGL-N could have SMAC-mimetic-like function in regulation of cell death and to enhance sensitivity of anticancer drugs.

\section{Materials and methods}

\section{Cell culture, antibodies, and reagents}

The MGL-proficient and deficient mouse embryonic fibroblasts (MEFs) were generated from our previous studies [2] and maintained as previously described [2]. MADT cell line was isolated from a tumor grown on the abdominal region of a $\mathrm{MGL}^{-1-}$ mouse (Supplementary Fig. 1) and maintained in DMEM with $10 \%$ fetal bovine serum (FBS). Human lung cancer cell lines A549 and H1299, and cervical cancer HeLa cell line were from the National Institutes of Health (NIH, National Cancer Institute (NCI)) and cultured in DMEM supplemented with $10 \%$ FBS. Human ovarian cancer cell lines OVCAR3 and SKOV3, were form ATCC, and MUM2C and C8161 [46] melanoma cells were kindly provided by Dr Brian J. Nickoloff, Loyola University Medical Center, Maywood, IL, USA. These cells were cultured in RPMI supplemented with $10 \%$ FBS. Cell lines used in this study were tested for mycoplasma contamination using the MycoFluor Mycoplasma Detection kit from Thermo Fisher (Waltham, MA, USA).

The XIAP and caspase-3 antibodies were from BD Transduction Laboratories (San Jose, CA, USA). The Phospho-AKT (Ser473), AKT, Cleaved PARP, cleaved caspase-3, cleaved caspase-8, pro-caspase-8 and 9 antibodies were from Cell Signaling (Danvers, MA, USA). The MGL antibody was generated by our laboratory and described in previous studies [1]. The GAPDH and vinculin antibodies were from Santa Cruz Biotechnologies (Dallas, TX, USA). The $\beta$-actin and $\alpha$-tubulin antibodies were from Sigma (St. Louis, MO, USA). The XIAP antibody for immunohistochemical staining was from Abcam (Cambridge, MA, USA). The MGL inhibitor URB602 was purchased from Sigma-Aldrich (St. Louis, MO, USA); it has IC50 $=28 \mu \mathrm{M}$ [47]. The other MGL inhibitor JZL184 was obtained from Cayman Chemical (Ann Arbor, MI, USA); it has $\mathrm{IC}_{50}=8 \mathrm{nM}$ [26]. Pancaspase inhibitor Z-VAD-FMK was purchased from R\&D Systems (Minneapolis, MN, USA). Cycloheximide was purchased from Sigma-Aldrich (St. Louis, MO, USA) and used $10 \mu \mathrm{g} / \mathrm{ml}$ for indicated times. Embelin was purchased from Cayman Chemical (Ann Arbor, MI, USA). Anticancer drugs doxorubicin, etoposide, and taxol were purchased from Sigma-Aldrich (St. Louis, MO, USA). The purified XIAP protein was purchased from R\&D Systems (Minneapolis, MN, USA). The purified S-HA-tag MGL protein was generated from the previous studies [1]. The matching human patient normal and tumor samples were from The Cooperative Human Tissue Network, an organization sponsored by the NCI. 


\section{MTT assays}

MTT cell proliferation assays were performed as previously described [1]. Briefly, cells seeded in a 96-well plate (for Figs. 3, 8a) or 12-well plate (for Fig. 8d) with indicated transfections or treatments, were incubated with $0.5 \mathrm{mg} / \mathrm{mL}$ MTT for $1 \mathrm{~h}$. The resulting formazan precipitate was dissolved in isopropanol with $0.04 \mathrm{~mol} / \mathrm{L} \mathrm{HCl}$. Absorbance was read with a Bio-Rad SmartSpec 3100 at $570 \mathrm{~nm}$ with background subtraction read at $690 \mathrm{~nm}$.

\section{Expression plasmids}

XIAP full-length and deletion variants vector were provided by Addgene (Watertown, MA, USA). pDsRedN1-MGL-WT vector (MGL-RFP) and pSR $\alpha$-HA-S-tagged MGL expression vector were previously described [1, 2]. The pDsRedN1MGL-S132A construct was generated using the pDsRedN1MGL-WT [2] as a template and the site-directed mutagenesis kit per protocol provided by the company (Agilent Technologies, Santa Clara, CA, USA). MGL-deletion variants were generated by subcloning the MGL-cDNA fragments generated by PCR into a pSR $\alpha$-HA-S-tag expression vector; primers for PCR-cloning as the followings: for MGL-1-60: 5'-ACAGCTAGCATGGAAACAGGACCTGAA-3' and 5'-C ATCCGCGGTCCATGGGACACA AAGAT-3'. For MGL1-105: 5'- ACAGCTAGCATGGAAACAGGACCTGAA-3' and 5'-CATCCGCGGAACGTGGAAGTCAGACAC 3'. For MGL-1-209: 5'-ACAGCTAGCATGG AAACAGGACC TGAA-3' and 5'-CATCCGCGGCAGGGGGTCTGAGTT ATA-3'. For MGL-106-209: 5'-ACAGCTAGCATGTTCG TCAGGGATGTG-3' and 5'-CATCCGCGGCAGGGG GTC TGAGTTATA 3'. For MGL-106-313: 5' ACAGCTAGCAT GTTCGTCAGGGATGTG-3' and 5'-CATCCGCGGGGGT GGGGACGCAGTTC-3'. For MGL-210-313: 5'-ACAGC TAGCATGATCTGCCGGGCAGGGC-3' and 5'-CATCCG CGGGGGTGGGGACGC AGTTC-3'. DNA sequencing was performed to confirm the integrity of MGL sequence for all expression vectors.

\section{Animal studies}

MGL-knockout mice were generated from our previous studies and maintained as described [2]. Animal studies were performed according to guidelines of the Institutional Animal Care and Use Committee of SUNY Upstate Medical University. Tissues used for histological studies were fixed in $4 \%$ paraformaldehyde and hematoxylin and eosin staining was performed as previously described and inspected by a pathologist (CC).

\section{Lentivirus-mediated knockdown of MGL}

Different nucleotide sequences targeting the human MGL are designed as follows: shRNA1 $5^{\prime}$-ccaggacaagactctcaagat-3'; shRNA2 5'-caactccgtcttccatgaaat-3'; shRNA3 5'ccaatcctgaatctgcaacaa- $3^{\prime}$. MGL knockdown was achieved by shRNA silencing as described previously [1]. The shRNA expression was mediated by lentiviruses that are prepared and used according to protocols from Addgene.

\section{Western blot analysis}

For western blotting analyses, cell lysate and tissue extraction were performed as described previously [1].

\section{Immunoprecipitation and S-tag pulldown assays}

Immunoprecipitation was performed as previously described [48, 49]. S-tag pulldown assay was performed using S-protein agarose beads (EMD Millipore, Billerica, MA). Approximately, $50 \mu \mathrm{l}$ of bead slurry was washed with buffer and mixed with cell lysate and the mixture was incubated overnight on a rotator at $4{ }^{\circ} \mathrm{C}$. The beads were then washed for three times and the associated proteins were denatured at $95^{\circ} \mathrm{C}$ in SDS-loading buffer, and subsequently analyzed by western blot analyses. Complete triton lysis buffer $(150 \mathrm{mM} \mathrm{NaCl}, 20 \mathrm{mM}$ HEPES, $1 \mathrm{mM}$ EDTA, $1 \%$ Triton-X 100, $1 \mathrm{mM}$ PMSF, $20 \mathrm{mM} \mathrm{NaF}, 20 \mathrm{mM} \mathrm{Na} \mathrm{VO}_{4}, 1 \%$ protease inhibitor cocktail (Sigma-Aldrich)) was used for making cell lysate, incubation, and washing.

\section{Statistical analysis}

Two-tail Student's $t$ test was used for statistical analysis. Tests are two-sided. Variance was similar between groups within each experiment.

Funding This work is supported by NIH grants CA121850 (YH) and DK62136 (YH), Michael Connolly Lung Cancer Research Grant, SUNY Upstate Medical University (YH), and Upstate Health Science Center Hendricks Intramural Research Grant (YH).

\section{Compliance with ethical standards}

Conflict of interest The authors declare that they have no conflict of interest.

Publisher's note Springer Nature remains neutral with regard to jurisdictional claims in published maps and institutional affiliations. 


\section{References}

1. Sun H, Jiang L, Luo X, Jin W, He Q, An J, et al. Potential tumor suppressive role of monoglyceride lipase in human colorectal cancer. Oncogene. 2013;32:234-41.

2. Liu R, Wang X, Curtiss C, Landas S, Rong R, Sheikh MS, et al. Monoglyceride lipase gene knockout in mice leads to increased incidence of lung adenocarcinoma. Cell Death Dis. 2018;9:36

3. Rajasekaran D, Jariwala N, Mendoza RG, Robertson CL, Akiel MA, Dozmorov M, et al. Staphylococcal nuclease and tudor domain containing 1 (SND1 protein) promotes hepatocarcinogenesis by inhibiting monoglyceride lipase (MGLL). J Biol Chem. 2016;291:10736-46.

4. Xiang W, Shi R, Kang X, Zhang X, Chen P, Zhang L, et al. Monoacylglycerol lipase regulates cannabinoid receptor 2dependent macrophage activation and cancer progression. Nat Commun. 2018;9:2574.

5. http://firebrowse.org/viewGene (accessed: 2019).

6. https://www.oncomine.org/resource/ (accessed: 2019).

7. Roos WP, Thomas AD, Kaina B. DNA damage and the balance between survival and death in cancer biology. Nat Rev Cancer. 2016;16:20-33.

8. Labi V, Erlacher M. How cell death shapes cancer. Cell Death Dis. 2015;6:e1675.

9. Baig S, Seevasant I, Mohamad J, Mukheem A, Huri HZ, Kamarul T. Potential of apoptotic pathway-targeted cancer therapeutic research: where do we stand? Cell Death Dis. 2016;7:e2058.

10. Eckelman BP, Salvesen GS, Scott FL. Human inhibitor of apoptosis proteins: why XIAP is the black sheep of the family. EMBO Rep. 2006;7:988-94.

11. Huang Y, Rich RL, Myszka DG, Wu H. Requirement of both the second and third BIR domains for the relief of X-linked inhibitor of apoptosis protein (XIAP)-mediated caspase inhibition by Smac. J Biol Chem. 2003;278:49517-22.

12. Tenev T, Zachariou A, Wilson R, Ditzel M, Meier P. IAPs are functionally non-equivalent and regulate effector caspases through distinct mechanisms. Nat Cell Biol. 2005;7:70-7.

13. Chai J, Shiozaki E, Srinivasula SM, Wu Q, Datta P, Alnemri ES, et al. Structural basis of caspase-7 inhibition by XIAP. Cell. 2001;104:769-80.

14. Silke J, Ekert PG, Day CL, Hawkins CJ, Baca M, Chew J, et al. Direct inhibition of caspase 3 is dispensable for the anti-apoptotic activity of XIAP. EMBO J. 2001;20:3114-23.

15. Riedl SJ, Fuentes P, Renatus M, Kairies N, Krapp S, Huber R, et al. Structural basis for the activation of human procaspase-7. Proc Natl Acad Sci USA. 2001;98:14790-5.

16. Suzuki Y, Nakabayashi Y, Takahashi R. Ubiquitin-protein ligase activity of $\mathrm{X}$-linked inhibitor of apoptosis protein promotes proteasomal degradation of caspase- 3 and enhances its anti-apoptotic effect in Fas-induced cell death. PNAS. 2001;98:8662-7.

17. Shiozaki EN, Chai J, Rigotti DJ, Riedl SJ, Li P, Srinivasula SM, et al. Mechanism of XIAP-mediated inhibition of caspase-9. Mol Cell. 2003;11:519-27.

18. Obexer P, Ausserlechner MJ. X-linked inhibitor of apoptosis protein - a critical death resistance regulator and therapeutic target for personalized cancer therapy. Front Oncol. 2014;4:197.

19. Riedl SJ, Renatus M, Schwarzenbacher R, Zhou Q, Sun C, Fesik SW, et al. Structural basis for the inhibition of caspase-3 by XIAP. Cell. 2001;104:791-800.

20. Scott F, Denault J, Riedl S, Shin H, Renatus M, Salvesen G. XIAP inhibits caspase- 3 and -7 using two binding sites: evolutionarily conserved mechanism of IAPs. EMBO J. 2005;24:645-55.

21. Galbán S, Duckett CS. XIAP as a ubiquitin ligase in cellular signaling. Cell Death Differ. 2010;17:54-60.
22. Samuels-Lev Y, O'Connor DJ, Bergamaschi D, Trigiante G, Hsieh JK, Zhong S, et al. ASPP proteins specifically stimulate the apoptotic function of p53. Mol Cell. 2001;8:781-94.

23. Rotem-Bamberger S, Katz C, Friedler A. Regulation of ASPP2 interaction with p53 core domain by an intramolecular autoinhibitory mechanism. PLoS ONE. 2013;8:e58470.

24. Liston P, Roy N, Tamai K, Lefebvre C, Baird S, Cherton-Horvat $\mathrm{G}$, et al. Suppression of apoptosis in mammalian cells by NAIP and a related family of IAP genes. Nature. 1996;379:349-53.

25. Deveraux Q, Takahashi R, Salvesen GS, Reed JC. X-linked IAP is a direct inhibitor of cell death proteases. Nature. 1997;388:300-30.

26. https://www.caymanchem.com/product/13158 (accessed 2020).

27. Nikolovska-Coleska Z, Xu L, Hu Z, Tomita Y, Li P, Roller PP, et al. Discovery of embelin as a cell-permeable, small-molecular weight inhibitor of XIAP through structure-based computational screening of a traditional herbal medicine three-dimensional structure database. J Med Chem. 2004;47:2430-40.

28. Nomura DK, Long JZ, Niessen S, Hoover HS, Ng S-W, Cravatt BF. Monoacylglycerol lipase resulates a fatty acid newwork that promotes cancer pathogenesis. Cell. 2010;140:49-61.

29. Lobry C, Oh P, Aifantis I. Oncogenic and tumor suppressor functions of Notch in cancer: it's NOTCH what you think. J Exp Med. 2011;208:1931-5.

30. Krisenko MO, Geahlen RL. Calling in SYK: SYK's dual role as a tumor promoter and tumor suppressor in cancer. Biochim Biophys Acta. 2015;1853:254-63.

31. Bosch-Presegué L, Vaquero A. The dual role of sirtuins in cancer. Genes Cancer. 2011;2:648-62.

32. Yang L, Han Y, Saiz FS, Minden MD. A tumor suppressor and oncogene: the WT1 story. Leukemia. 2007;21:868-76.

33. de Caestecker MP, Ester Piek E, Anita B, Roberts AB. Role of transforming growth factor-beta signaling in cancer. J Natl Cancer Inst. 2000;92:1388-402

34. Nakatani Y, Kleffmann T, Linke K, Condon S, Hinds M, Day C. Regulation of ubiquitin transfer by XIAP, a dimeric RING E3 ligase. Biochem J. 2013;450:629-38.

35. Yang Y, Fang S, Jensen JP, Weissman AM, Ashwell JD. Ubiquitin protein ligase activity of IAPs and their degradation in proteasomes in response to apoptotic stimuli. Science. 2000;288:874-7.

36. Lotocki G, Alonso OF, Frydel B, Dietrich WD, Keane RW. Monoubiquitination and cellular distribution of XIAP in neurons after traumatic brain injury. J Cereb Blood Flow Metab. 2003;23:1129-36.

37. Xia Y, Pao GM, Chen HW, Verma IM, Hunter T. Enhancement of BRCA1 E3 ubiquitin ligase activity through direct interaction with the BARD1 protein. J Biol Chem. 2003;278:5255-63.

38. Labar G, Bauvois C, Borel F, Ferrer JL, Wouters J, Lambert DM Crystal structure of the human monoacylglycerol lipase, a key actor in endocannabinoid signaling. Chembiochem. 2010;11:218-27.

39. Bertrand T, Augé F, Houtmann J, Rak A, Vallée F, Mikol V, et al. Structural basis for human monoglyceride lipase inhibition. J Mol Biol. 2010;396:663-73.

40. Chai J, Du C, Wu JW, Kyin S, Wang X, Shi Y. Structural and biochemical basis of apoptotic activation by Smac/DIABLO. Nature. 2000;406:855-62.

41. Flanagan L, Sebastià J, Tuffy LP, Spring A, Lichawska A, Devocelle M, et al. XIAP impairs Smac release from the mitochondria during apoptosis. Cell Death Dis. 2010;1:e49.

42. Schimmer AD, Dalili S, Batey RA, Riedl SJ. Targeting XIAP for the treatment of malignancy. Cell Death Differ. 2006;13:179-88.

43. Krepela E, Dankova P, Moravcikova E, Krepelova A, Prochazka $\mathrm{J}$, Cermak J, et al. Increased expression of inhibitor of apoptosis 
proteins, survivin and XIAP, in non-small cell lung carcinoma. Int J Oncol. 2009;35:1449-62.

44. Shiraki K, Sugimoto K, Yamanaka Y, Yamaguchi Y, Saitou Y, Ito $\mathrm{K}$, et al. Overexpression of $\mathrm{X}$-linked inhibitor of apoptosis in human hepatocellular carcinoma. Int J Mol Med. 2003;12:705-8.

45. https://www.cancer.gov/about-cancer/treatment/clinical-trials/ intervention/smac-mimetic-lcl161 (accessed: 2019).

46. Nickoloff BJ, Hendrix MJ, Pollock PM, Trent JM, Miele L, Qin JZ. Notch and NOXA-related pathways in melanoma cells. J Investig Dermatol Symp Proc. 2005;10:95-104.
47. https://www.sigmaaldrich.com/catalog/product/sigma/u3010?lan$\mathrm{g}=\mathrm{en}^{\circledR}$ ion $=$ US (accessed: 2020 ).

48. Montalbano J, Lui K, Sheikh MS, Huang Y. Identification and characterization of RBEL1 subfamily of GTPases in the Ras superfamily involved in cell growth regulation. J Biol Chem. 2009;284:18129-42.

49. Rong R, Jiang LY, Sheikh MS, Huang Y. Mitotic kinase AuroraA phosphorylates RASSF1A and modulates RASSF1A-mediated microtubule interaction and M-phase cell cycle regulation. Oncogene. 2007;26:7700-8. 